Förderschwerpunkt Palliativmedizin

\section{Begleitevaluation der Deutschen Krebshilfe}

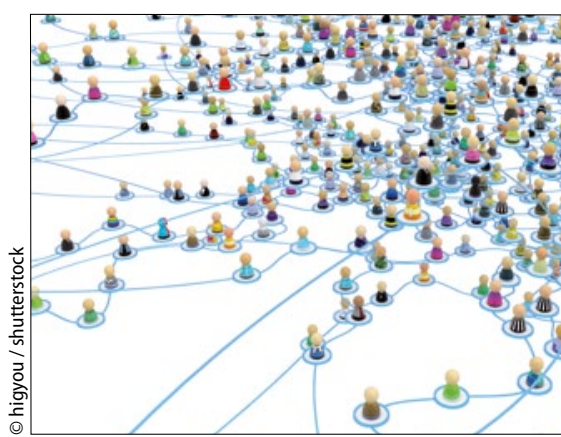

Viele Projekte möchten durch Beratung und Koordination die ambulante Versorgung von Palliativpatienten verbessern.

— Ein Ziel der Deutschen Krebshilfe ist es, die ambulante Versorgung von Palliativpatienten zu optimieren. In einer Begleitevaluation ihres Förderschwerpunkts Palliativmedizin wurden zwölf Projekte erfasst, die diesen Vorsatz verfolgen. Im Zeitraum von 2009 bis 2011 konnten die Daten von insgesamt 2.663 Patienten ausgewertet werden [Schmerz 2011;25(6):676-84]. Zu Beginn und Ende der Behandlung sowie bei einem Wechsel des betreuenden Palliativdienstes (etwa bei einem Übergang von einer stationären Behandlung zur Betreuung durch einen ambulanten Pflegedienst) wurden bei ihnen der Basisbogen der Hospiz- und Palliativerfassung (HOPE), das Minimale Dokumentationssystem für Palliativpatienten (MIDOS) und der Barthel-Index dokumentiert.

Alle Projekte fokussierten auf Beratung und Koordination, in einigen Fällen auch auf Case Management. Beschreibungen zufolge hal- fen die Projekte besonders, die Leistungen zu verbessern und auszuweiten, zum Beispiel ermöglichten sie die Betreuung von mehr Patienten. Zusätzlich konnten neue Versorgungsangebote eingeführt und Krankenhausaufenthalte vermieden werden.

Misstrauen, Konkurrenzdenken und Unverständnis anderer Versorger, vor allem von Pflegediensten und Hausärzten, wurden als wesentliche Probleme für die Umsetzung angegeben. Da sich die Projekte bezüglich

\section{Selektivverträge}

— Ärzte können künftig bedenkenlos über private Abrechnungsstellen oder Ärzteverbände ihre Leistungen aus Selektivverträgen abrechnen. Möglich macht dies eine Erweiterung des fünften Sozialgesetzbuchs (SGB V). Bundestag und Bundesrat haben den neuen § 295a zusammen mit dem Änderungsantrag zum Infektionsschutzgesetz abgesegnet. Nach seiner Veröffentlichung im Bundesgesetzblatt Anfang August ist er offiziell in Kraft.

In der Neuregelung wird explizit klargestellt, dass Ärzte für die Abrechnung von Leistungen innerhalb von Verträgen nach §§ $73 \mathrm{~b}, 73 \mathrm{c}$ oder $140 \mathrm{a}$ SGB V erforderliche Daten an den Vertragspartner auf Leistungserbrin- der behandelten Patientengruppe sowie der durchgeführten Interventionen deutlich unterschieden, waren durch die Auswertung keine generalisierbaren Rückschlüsse möglich. Die Frage, ob das Projekt etwas am Versorgungsalltag geändert habe wurde allerdings durchgehend positiv beantwortet. Hilfreich sind die Ergebnisse für die Entwicklung der ambulanten Palliativversorgung. In der aktuellen Diskussion können sie die Beleuchtung des Zusammenhangs von Versorgungsstrukturen, Patientenzielgruppe, dokumentierten Daten und Behandlungsergebnissen unterstützen, so das Fazit der Autoren.

Kim Jené

\title{
Abrechnung: Ärzte dürfen Patientendaten weitergeben
}

gerseite oder an eine von diesem beauftragte Stelle übermitteln dürfen. Darüber hinaus heißt es in Absatz 2 des neuen Paragrafen, dass auch die Beauftragung einer nicht öffentlichen Stelle "zulässig ist".

Allerdings müssen die Patienten künftig bereits bei der Einschreibung in einen Selektivvertrag umfassend über die vorgesehene Datenübermittlung informiert werden, und sie müssen ihre schriftliche Einwilligung geben. Bei Patienten, die bereits in Selektivverträge eingeschrieben sind, ist die Einwilligung - sofern sie nicht bereits Bestandteil der Einschreibung war - gegebenenfalls nachzuholen.

Rebekka Höh

\section{Update der EAPC-Leitlinie \\ Opioide gegen Tumorschmerzen}

— Die European Association for Palliative Care (EAPC) aktualisierte ihre Leitlinie zum Gebrauch von Opioiden in der Behandlung von Tumorschmerzen [Lancet Oncol. 2012;13(2):e58-e68]. Formuliert wurden 16 evidenzbasierte Empfehlungen unter Berücksichtigung anderer aktueller Leitlinien und der Ergebnisse randomisierter kontrollierter Studien. Bei der Entwicklung des Updates legten Augusto Caraceni und Kollegen das
GRADE (Grading of Recommendations Assessment, Development and Evaluation)-System zugrunde. Thematisiert werden unter anderem Opioide der WHO-Stufen II und III sowie der Gebrauch von Opioiden für die Behandlung von Durchbruchschmerzen. Auch die Therapie Opioid-bezogener Emesis und Obstipation finden Beachtung. Zuletzt war die ursprünglich 1996 publizierte Leitlinie 2001 aktualisiert worden. Die Wissenschaftler be- schreiben, dass bisher harte Evidenz aus randomisierten kontrollierten Studien fehlt. Opioide sollten den Autoren zufolge zwar eigentlich durch Spezialisten verschrieben werden, Realität sei aber, dass dies häufig durch unspezialisierte praktische Ärzte geschehe. Sie hoffen, dass dieser Expertenkonsens mit klinischen Empfehlungen für die Therapie von Tumorschmerzen hilfreich ist und Patienten von ihm profitieren werden. Kim Jené 